LINGÜÍSTICA 



\title{
LA ENSEÑANZA DE LA EXPRESIÓN ESCRITA EN EL MÉTODO COMUNICATIVO MEDIANTE EL ENFOQUE AL PROCESO
}

\author{
Marta Madrigal Abarca
}

\begin{abstract}
RESUMEN
El objetivo de este artículo es mostrar cómo las actividades que se realizan en las fases de la preescritura, el borrador y la revisión del Enfoque al proceso se pueden integrar a las estrategias del Método comunicativo para llevar a cabo el ejercicio de la escritura de manera más eficaz. Este método, al integrar las cuatro destrezas, permite un espacio abierto y multidisciplinario para que la enseñanza de la expresión escrita sea más dinámica e interesante para los estudiantes, pues éstos comprenden que el propósito está en la comunicación, en los pasos que se desarrollan para lograr esa comunicación y no tanto en el producto final.

Palabras clave: Método comunicativo, Enfoque al proceso, preescritura, borrador, revisión.
\end{abstract}

\begin{abstract}
The objective of this article is to show how the activities that are realized in the prewriting stages (draft and revision of the process focus) can be integrated into the Communicative Method to bring the writing exercise to a more efficient conclusion. This method, upon integrating the four skills, will permit multiple disciplines and room for the teaching of the written expression that will be more dynamic and interesting for the students. This is because they will understand that the purpose is in the communication and the development steps taken to achieve that communication and not so much in the final product.
\end{abstract}

Key words: Communicative Method, Process Focus, prewriting, draft, revision.

\section{Introducción}

\subsection{Los métodos en la enseñanza de idiomas}

En los últimos años, se han originado muy diversos métodos para la enseñanza de una L2, sin embargo, no hay una uniformidad de criterios para decidir cuál es el más

M.L. Marta Madrigal Abarca. Magíster en Español como Segunda Lengua. Profesora de la Escuela de Filología, Lingüística y Literatura de la Universidad de Costa Rica, San José, Costa Rica.

Correo electrónico: madrigal_ma@yahoo.com.mx

Recepción: 27- 10- 2009

Aceptación: 11- 12- 2009 
conveniente o cuál se ajusta más a la tarea de la enseñanza. Para Richards y Rodgers (1998), esta proliferación de enfoques es de gran utilidad para el profesor y coordinador de programas por la amplia variedad de modelos y materiales que se ofrecen, pero a veces confunden, ya que los métodos parecen estar basados en diferentes puntos de vista de lo que es la lengua y de cómo se aprende.

Es claro que el origen de todos estos métodos ha tenido, entre otras fuentes, la teoría lingüística vigente en cada época, la cual era imperante en los centros académicos y, desde ahí, transmitida al colectivo de profesores de idiomas. Estas implicaciones teóricas han aglutinado investigaciones y concepciones del concepto de lengua y se cuestionan sobre cómo se desarrolla el proceso de aprendizaje, cómo se puede enseñar mejor, o cómo se puede lograr un mayor grado de eficacia en la docencia.

Para afrontar estas preguntas, el profesor, de acuerdo con los objetivos que se plantee, debe tomar en cuenta una metodología que le facilite los instrumentos prácticos para la enseñanza. Según lo señala Aquilino Sánchez "Utilizar una metodología en la clase de idiomas equivale a elegir un camino determinado para la docencia, frente a otros posibles a que el profesor tiene opción" (1997:23).

El hecho lingüístico es muy complejo. Consecuentemente, es difícil concebir una metodología sin tener en cuenta el objeto que se pretende enseñar, es decir, la lengua. La relación de esta con la teoría como punto de partida, junto con los objetivos que se proponen para la enseñanza son los constituyentes esenciales en la formación metodológica y los ejes fundamentales en el proceso de aprendizaje. El método, por lo tanto, debe necesariamente concretarse en la práctica a través de operaciones, de las que se pueden deducir cuáles son los principios o teoría de los que se parte, cuáles son los objetivos que se proponen y cuáles técnicas van a ayudar a lograr los objetivos. De igual forma, debe materializarse a través de actividades concretas, las cuales son necesarias para alcanzar los fines propuestos.

En la mayoría de los casos, el profesor de idiomas está consciente de que, al enseñar una lengua, debe elegir el camino más propicio y eficaz para lograr que sus estudiantes aprendan. Estos últimos no constituyen un grupo homogéneo, es decir, con las mismas habilidades ni con los mismos estilos cognitivos de aprendizaje. Muchas veces, de acuerdo con las características de cada uno de los aprendices, el profesor debe seleccionar el método que más se ajuste al grupo en total o el que esté más acorde con los objetivos que se propone enseñar. No obstante, sea cual sea el método seleccionado debe cubrir las necesidades del estudiante, tanto como los propósitos del profesor.

\section{El Método comunicativo}

La metodología comunicativa está estrechamente relacionada con los programas nocionales-funcionales, así como con los de la pragmática. Los primeros definían el contenido y reorientaban los criterios de selección, los cuales responden a una base estructural. La pragmática, por su parte, pretende ser un punto de balance entre los valores del estructuralismo y del generativismo.

Dado que la complejidad lingüística solo es observable en el uso de la lengua y no en la formalización teórica abstraída de la realidad, los estudios lingüísticos se concentran más en el habla que en el lenguaje. Así, el uso lingüístico y el habla tienen como un único punto de referencia, el acto de comunicación por medio de un sistema lingüístico concreto. Por este 
motivo, la pragmática se equipara, de alguna manera, con el paradigma de la comunicación; a su vez, la metodología comunicativa queda integrada al paradigma de la pragmática. Los pilares fundamentales de tales concepciones se derivan de:

a. El lenguaje se estudia como algo globalizado, ya sea como discurso o como texto.

b. El lenguaje no se presenta en primera instancia como un sistema, sino como un medio que sirve o propicia la comunicación. Por tal motivo, no interesa de manera prioritaria el análisis de las formas, sino el proceso comunicativo y las relaciones entre los sujetos que interactúan.

c. La lengua, al ser analizada desde la perspectiva del uso, es un instrumento con funciones comunicativas determinadas. Fuera del ámbito del uso carece de funcionalidad y de sentido.

d. Se deja de lado la noción de lenguaje ideal para centrarse en el estudio del lenguaje real.

\subsection{Las características de la comunicación}

La comunicación se da mediante el lenguaje; se trata de decir algo a alguien, ya sea oralmente o por escrito. El énfasis, por tanto, está en el contenido. De esta manera, el proceso comunicativo nunca se da aisladamente, sino dentro de un contexto o situación. Dicho contexto contribuye al proceso, facilitando la comprensión de los mensajes intercambiados, por medio de elementos extralingüísticos que lo distinguen.

La comunicación se establece siempre por alguna razón o interés, lo cual significa que un proceso comunicativo nunca se inicia sin motivaciones reales. De allí que la comunicación real esté relacionada con la consecución de un objetivo mediante la realización de un trabajo o tarea; es decir, prevalece lo práctico (operativo) ante lo abstracto. Asimismo, el proceso comunicativo tiene lugar porque los interlocutores se atienen al código de señales o signos preestablecidos. En este contexto, la gramática se concibe como un medio para lograr los fines comunicativos, pero no como un fin en sí mismo.

\subsection{Las actividades comunicativas}

Las actividades en el marco comunicativo poseen características específicas, es decir, deben estar en concordancia con las particularidades del lenguaje, de tal forma que se fundamentan, principalmente, en la transmisión de contenido relevante para los interlocutores. Es claro entonces que estas actividades subordinan la forma (aspectos formales de la lengua, la gramática) al contenido (mensaje que se desea trasmitir) y son muy interactivas, ya que los participantes necesitan desarrollar una acción concreta y no abstracta, por ejemplo, solucionar un problema más que explicar una regla gramatical.

\subsection{La selección del contenido comunicativo}

Puesto que el objetivo primordial es que los estudiantes se comuniquen, es necesario definir previamente los campos semánticos y áreas temáticas, así como las situaciones en las que se desarrollará la comunicación, las funciones concretas que se deben realizar y los registros lingüísticos (estructuras, léxico) implicados en tales funciones. Además, se deben tomar en cuenta otros elementos como la selección de los contextos o situaciones dentro de 
los cuales se llevan a cabo determinadas funciones lingüísticas, las estructuras o exponentes lingüísticos que posibilitan la actualización de esas mismas funciones lingüísticas, el vocabulario necesario para desarrollar tales funciones en situaciones o contextos descritos, la identificación de los elementos morfológicos y las reglas sintácticas en la expresión verbal y escrita para que la comunicación sea eficaz y no ambigua, los elementos fonéticos de la lengua oral en un contexto comunicativo, los sonidos dentro de la cadena hablada, tanto en la escucha como en el habla y los problemas semánticos derivados de los factores no lingüísticos en relación con el contexto de los estudiantes.

\subsection{Un método multidisciplinario}

La exigencia del Método comunicativo deriva de la complejidad misma del proceso de la comunicación y del lenguaje que lo propicia, en consecuencia, no se deben obviar los elementos primordiales para que la comunicación se concrete. De tal manera que, si las funciones y las situaciones son parámetros fundamentales que constituyen el punto de partida para decidir y organizar el contenido, tampoco puede dejarse de lado el código gramatical que, aunque esté subordinado en las situaciones comunicativas, es necesario para que se dé una comunicación eficaz.

Asimismo, en este método se desarrollan tanto las destrezas interpretativas (la escucha y la lectura) como las expresivas (el habla y la escritura). Este hecho es sumamente importante, ya que la comunicación no solo se da en él ámbito del habla y la escucha, sino también en el área de la escritura. Por lo tanto, la multiplicidad se presenta en los materiales y actividades que incluyen las cuatro destrezas, así como en los contextos y las situaciones en que se da la comunicación. Debido a esta condición disciplinaria, los investigadores y profesores de idiomas han llegado a pensar que no existe un método, sino varios métodos comunicativos y, en algunos casos, han aducido también que esta metodología, más que un método, es un enfoque. Esta diferenciación no es necesaria, pues como lo manifiesta Aquilino Sánchez:

Es verdad que el método comunicativo admite más posibilidades de concreción debido a su variedad.

Pero si conserva la coherencia y la cohesión entre los elementos que lo integran, no hay necesidad de establecer varios compartimentos estanco. (Sánchez 1997:212)

En última instancia, de acuerdo con los postulados de esta metodología así como con los criterios de Sánchez, este tipo de enseñanza comunicativa de la lengua se puede denominar Método comunicativo.

\section{El Método comunicativo y la escritura}

Aunque este método se centra en las habilidades orales en el aprendizaje de un idioma, puede aplicarse perfectamente a la enseñanza de la composición de una segunda lengua, ya que introduce la lengua escrita desde el inicio del aprendizaje, no solo como una destreza para reforzar lo aprendido de forma oral, sino como una habilidad que tiene técnicas y objetivos propios.

Entre muchas de las ventajas que ofrece, está la de brindar un esquema claro y detallado de organización de las actividades que se realizarán tanto dentro como fuera del aula. Además, tiene el objetivo de poner en contacto al estudiante con el uso lingüístico que aprenderá. En otras palabras, es vital que el aprendiz tome conciencia de qué es lo que tiene que aprender y qué lo que tendrá que saber hacer al final de cada trabajo. También dirige la 
atención del aprendiz hacia la comprensión del significado del uso y no tanto de la forma, por medio de contextos reales: desde fotografías hasta biografías de personajes interesantes; desde informaciones tomadas de la prensa hasta datos reales de los estudiantes de la clase. Cabe recordar también que la escritura tiene que estar integrada a las otras destrezas. Esta integración tiene varios propósitos:

a. Permite la práctica de los mismos contenidos lingüísticos o funcionales en las distintas destrezas.

b. Desarrolla dos o más destrezas lingüísticas dentro de un mismo contexto.

c. Aproxima el uso de la lengua a la vida real.

\section{El Método comunicativo y el Enfoque al proceso}

Dadas las características del Método comunicativo, este se constituye en un espacio abierto y óptimo para enseñar la destreza de la escritura usando el Enfoque al proceso, ya que las actividades realizadas en cada una de las etapas del proceso (preescritura, borrador y revisión) se ajustan a las actividades que se pueden realizar en el Método comunicativo.

\section{1. $\quad$ El enfoque basado en el proceso}

Los modelos cognitivos han generado variados y distintos enfoques metodológicos para la enseñanza de la expresión escrita, los cuales pretenden que el estudiante aprenda a expresarse, mediante la escritura, de una manera más eficaz. Entre los enfoques más renombrados se encuentra el enfoque basado en el proceso.

Según algunos investigadores de la expresión escrita, el producto final obtenido al terminar de escribir un texto es importante, pero también lo es el proceso por medio del cual se llegó a ese producto final. Sin embargo, en muchos casos, ese proceso no es tomado en cuenta. Al respecto, Brookes y Grundy expresan:

\footnotetext{
A todos nosotros (y esto incluye a nuestros estudiantes) nos gustaría elaborar productos finales que sean imaginativos, seguros, personales y públicos fluidos y sin errores. Algunos maestros les piden a sus estudiantes crear una serie de productos y esperan que la repetición constante ayudada por el cuidadoso señalamiento y evaluación resultarán eventualmente en un aceptable producto no siempre dándose cuenta de cuan interesante y efectivo puede ser el proceso. (Brookes y Grundy 1998:15)
}

Debido a esta situación, muchos profesores prefieren abordar la expresión escrita con un modelo que enfatice el aspecto cognitivo como lo es el Enfoque al proceso, cuyo eje central gira en torno a las distintas actividades de pensamiento superior que realiza un autor para componer un escrito, desde donde se crea una circunstancia social que exige producir un texto hasta darlo por acabado. De este modo, el texto no es visto como un producto terminado, sino como un proceso, el cual involucra tanto los elementos que proceden del exterior como las circunstancias que motivan o inducen a escribir, es decir, los elementos específicos que se dan en el interior de la mente del escritor. Así, es importante enseñar no solo cómo debe ser la versión final de un escrito, sino mostrar y aprender todos los pasos intermedios y las estrategias que deben utilizarse durante el proceso de creación y redacción.

En consecuencia, en una clase de composición, el énfasis debe estar en el alumno y no en el texto que escribe, ya que se pretende que el estudiante piense, haga esquemas, ordene 
ideas, pula la estructura de las frases y revise el escrito. En fin, lo relevante es que, al final del curso, el estudiante esté consciente de los pasos que sigue al escribir y no tanto de que sus textos sean absolutamente correctos.

\subsubsection{Las fases en el Enfoque al proceso}

Flower y Hayes (Cassany, 1989:145) conciben la composición como una acción dirigida a la consecución de objetivos retóricos, por lo cual proponen tres etapas básicas: la planificación, la redacción y la revisión, que a su vez dan origen a otras sub-etapas como la generación de ideas, la formulación de objetivos y la evaluación de producciones intermedias, entre otros. Para cada una de estas etapas, se enseñan varias técnicas útiles para la redacción. Asimismo, Murray (Richards 1990:108) distingue tres fases: preescritura, borrador y revisión.

La primera fase involucra la búsqueda de temas, mediante la obtención de ideas sobre el tema y cómo pueden interactuar entre sí, además de cómo se desarrollan y se organizan, tomando en cuenta también la audiencia y el propósito. La segunda fase se refiere al borrador, es decir, a escribir de manera preliminar, las ideas obtenidas en la etapa de preescritura. El escritor bosqueja una idea, la examina y la continúa durante algún tiempo, a veces dejándola seguir su propio curso. Lo escrito sirve para generar nuevas ideas, planes y metas. Así, el proceso de escritura crea su propio significado. El escritor puede regresar a la fase del borrador $\mathrm{y}$ alternar entre las fases de hacer el borrador y hacer el bosquejo.

La última fase involucra la evaluación de lo escrito. En este estadio, se eliminan o se añaden elementos para ayudarle a la escritura a decir lo que intenta decir. Aunque se considera la última fase, la revisión también puede realizarse en cualquier momento del proceso y no solo considerarla como etapa final.

De esta manera, lo desarrollado en estas tres etapas, programa el conjunto de estrategias o habilidades y actitudes respecto de lo escrito. Según este enfoque, estas estrategias y actitudes son las que se deben enseñar para que el escritor competente elabore su trabajo de una manera más eficiente. Se trata de procesos mentales que incluyen muy diversas tareas, las cuales el escritor debe plantearse durante todo el proceso de la composición.

En resumen, el Enfoque al proceso pretende enseñar al alumno a pensar en lo que va a decir, para quién o quiénes va a escribir y cómo empezar. Entre otras actividades, debe aprender a seleccionar el punto principal de cada fragmento, decidir el orden en el cual se van a desarrollar las ideas y los párrafos y elegir un título. Una vez escrito el primer borrador, debe estar seguro de la escritura correcta, así como del uso de las letras mayúsculas, de los signos de puntuación y de la ortografía. Asimismo, debe saber escoger las palabras que tengan significados convenientes al contexto y evitar tanto el uso de palabras e ideas ambiguas como de cacofonías y repeticiones. Para atraer la atención del lector, tendrá que producir suficientes puntos de interés e ilustrarlos para que sean más comprensibles, así como referirse a las ideas de otros. Finalmente, debe corregir las pruebas, desde los errores mecánicos más pequeños como la ortografía hasta el contenido de todos los párrafos del escrito.

\subsubsection{Las actividades de enseñanza en el Enfoque al proceso relacionadas con el Método comunicativo}

Como el Método comunicativo debe incluir las cuatro destrezas, las actividades se vuelven muy interactivas. Sin embargo, las actividades desarrolladas en el Enfoque al proceso 
pueden ser diseñadas de igual manera, de modo que el estudiante sea tan participativo que convierta el espacio de la escritura en un momento agradable y divertido. Es necesario recordar que para muchos estudiantes, el acto de escribir es difícil y aburrido, pero esto es porque no le han enseñado que al integrarse las cuatro destrezas, se da una mayor interacción entre todos los participantes, lo cual hace de la escritura un espacio muy entretenido.

\subsection{Actividades de la fase de preescritura}

Las tareas en esta fase son diseñadas para ayudar a los aprendices a generar planes y desarrollar ideas para la redacción. También funcionan como un mecanismo de estímulo inicial al proceso de escritura. Es decir, proveen la motivación que el estudiante necesita para empezar a escribir, por ejemplo:

a. Los diarios: los estudiantes exploran ideas y graban pensamientos en el diario.

b. Lluvia de ideas: en grupo o individualmente, los aprendices intercambian rápidamente información sobre el tema o sobre algo que ellos han seleccionado para leer.

c. Asociación libre: se escribe un tema en la pizarra, luego los estudiantes dicen cualquier palabra que les venga a la mente cuando ellos leen el tema de la pizarra.

d. Valores de clarificación: se comparan las actitudes hacia una variedad de problemas y situaciones específicas.

e. Mapa de palabras: se escribe un tema y se organizan las palabras y conceptos relacionados.

f. Escritura rápida: los estudiantes escriben sobre un tema tanto como ellos puedan en un tiempo dado, sin preocuparse de la forma.

g. Actividades para reunir información: se les da a los estudiantes una asignación relacionada con un tema para que busquen la información por medio de entrevistas, encuestas, trabajo de campo, entre otros. También se pueden incluir canciones, programas radiales, programas de televisión y artículos periodísticos; de esta manera, se acerca al estudiante al contexto real, lo cual es uno de los puntos fundamentales del Método comunicativo.

\subsection{Actividades de la fase del borrador}

En esta fase, el escritor pasa del intento inicial del bosquejo de las diferentes secciones de la redacción hacia el borrador completo del párrafo o del ensayo. El escritor bosqueja algunas de las ideas generadas en la fase de preescritura, pero las desarrolla y las elabora en este estadio. Algunas de las actividades que se proponen son:

a. Preguntas estratégicas: los estudiantes examinan un grupo de preguntas que los ayudan a enfocar, priorizar y seleccionar ideas de escritura; por ejemplo: ¿qué quiere escribir realmente?, ¿cuál es la meta?, ¿cuál es la actitud hacia esa tarea?, ¿qué sabe sobre ese tema?, ¿qué necesita todavía averiguar del tema?, ¿cuáles ideas parecen quedar juntas?, ¿qué es lo más importante del tema?, ¿quién quisiera leer lo que se va a escribir?

b. Escritura con énfasis en el tiempo: se escribe rápidamente, en un período de tiempo específico, sobre un tema seleccionado por los estudiantes en la fase de la preescritura.

c. Elaboración de ejercicios: se reescribe un párrafo complejo con oraciones más simples. 
d. Párrafo desordenado: se reorganiza un párrafo a partir de una serie de oraciones presentadas de manera desordenada.

e. Ensayo desordenado: los estudiantes tienen un grupo de párrafos que deben juntar hasta obtener el ensayo completo.

f. Redacción de la tesis y el tema: a partir de algunas frases se desarrollan la tesis y el tema.

g. Escritura rápida: los estudiantes escriben rápidamente varias secciones de una composición (el principio, la parte central y la conclusión).

h. Borrador en grupo: los estudiantes trabajan juntos en la elaboración del borrador de las diferentes partes de una composición.

\subsection{Actividades de la fase de la revisión}

Las actividades en esta etapa corresponden a la edición y la corrección de pruebas, las cuales incluyen:

a. Retroalimentación entre compañeros: los estudiantes trabajan en grupos y leen, critican y corrigen pruebas de sus propias composiciones.

b. Actividades de corrección en grupo: se suprimen ciertos elementos como oraciones que constituyen temas y tesis, así como conectores y los estudiantes deben agregar el elemento suprimido.

c. Ejercicios de reescritura: se distribuyen oraciones no refinadas y párrafos confusos y los estudiantes deben reescribir el ensayo.

d. Retroalimentación del profesor: esto puede darse en diferentes estadios durante el proceso de escritura, no solo al final.

e. Chequeo de listas: los estudiantes tienen cortas listas para chequear, poner atención a algunos rasgos específicos de la frase, párrafo u organización del texto que ellos deberían atender en su revisión.

\section{Conclusión}

Aunque el Método comunicativo enfatiza las habilidades orales en el aprendizaje de un idioma, puede aplicarse perfectamente a la enseñanza de la composición de una segunda lengua, pues introduce la lengua escrita desde el inicio del aprendizaje, no solo como una destreza para reforzar lo aprendido de forma oral, sino como una habilidad con técnicas y objetivos propios.

Asimismo, el Método comunicativo intenta integrar las cuatro destrezas mediante muy diversas actividades, las cuales pueden entroncar de una manera muy especial con las actividades relacionadas con el Enfoque al proceso. De manera que, a pesar de que el acto de escribir es individual, en el proceso mediante el cual se llega a la finalidad del producto, el estudiante no solo escribe, sino que lee, escucha y habla; esto hace que la experiencia de la redacción de un texto sea compartida por todos los integrantes de la clase. Es decir, el Enfoque al proceso para la enseñanza de la expresión escrita dentro del marco del Método comunicativo se vuelve más interesante y enriquecedor al relacionar la destreza de la escritura con las otras destrezas.

Para concluir, se puede afirmar que una clase de composición diseñada de este modo es muy atractiva y eficaz para los estudiantes que desean aprender la lengua meta de manera integral. 
MADRIGAL: La enseñanza de la expresión escrita en el Método comunicativo mediante...

\section{Bibliografía}

Brookes, Arthur y P. Grundy. 1998. Beginnig to write: writing activities for elementary and intermediate learners. Cambridge: Cambridge University Press.

Brown, Douglas. 1994. Teaching by Principles: an interactive approach to language pedagogy. New Jersey: Prentice Hall Regents.

Brown, Douglas. 2000. Principles of Language Learning and Teaching. New York: Addison Wesley Longman, Inc.

Carduner, Jessie. 2002. "Using Classroom Assessment Techniques to Improve Foreign Language Composition Courses". Foreign Language Annals, 35 (5): pp

Cassany, Daniel. 1989. Describir el escribir. España: Ediciones Paidós Ibérica, S.A.

1999. Construir la escritura. España: Ediciones Paidós Ibérica, S.A.

2000. Reparar la escritura. Didáctica de la corrección de lo escrito. Barcelona: Editorial Graó.

Condemarín, Mabel y Chadwick, Mariana. 1999. Taller de Escritura. Santiago de Chile: Editorial Universitaria, S.A.

Ellis, Rod. 1997. SLA Research and Language Teaching. Oxford: Oxford University Press.

Encina, Alonso. 1986. ¿Cómo ser profesorla y querer seguir siéndolo? Madrid: Edelsa Grupo Didascalia, S.A.

Gerrard, Lisa y Sheri Spaine Long. 1993. Redacción y revisión. United States of America: McGraw-Hill, Inc.

Harmer, Jeremy. 1987. Teaching and learning grammar. New York: Longman Publishing.

Jurado Spuch, Adelaida. 2005. "Writing activities should be as much concerned with process as with product". En: Actas II, IX Simposio Internacional de Comunicación Social. Centro de Lingüística Aplicada. Santiago de Cuba

Larsen-Freeman. 1986. Techniques and principles in language teaching. Oxfor: Oxford University Press.

Llobera, M. 1995. Competencia comunicativa. Documentos básicos en la enseñanza de lenguas extranjeras. Madrid:Edelsa Grupo Didascalia, S.A. 
Madrigal Abarca, Marta. 2005. "La enseñanza de la escritura como proceso a estudiantes de español como segunda lengua de nivel avanzado". Informe final de práctica dirigida. Universidad de Costa Rica.

Manchón, R., Roca, J. y Murphy, L. 2000. "La influencia de la variable <grado de dominio de la L2> en los procesos de composición en lengua extranjera: hallazgos recientes de investigación”. En: Muñoz, Carmen (compiladora)

Nance K, y Rivera, I. 1996. Técnicas de composición. Massachussets: D.C. Heath and Company.

Navarro Thames, Carlos. 1998. “Técnicas para la corrección de errores del habla en cursos de idiomas". Kañina 22 (2): 103-112.

Nunan, David. 1999. Second Language Teaching and Learning. Boston: Heinkle \& Heinkle Publisehers.

Omaggio Hadley, Alice. 1993 Teaching Language in Context. Boston: Heinle \& Heinle Publishers.

Richards, Jack C. y T. Rodgers. 1998. Enfoques y métodos en la enseñanza de idiomas. Cambridge: Cambridge University Press.

Richards, Jack C. 1990. The Language Matrix. Cambridge: Cambridge University Press.

2001. Curriculum development in language teaching. Cambridge: Cambridge University Press.

Sánchez Pérez, Aquilino. 1997. Los métodos en la enseñanza de idiomas. España: Sociedad General Española de Librería S.A.

Solís, Mayra. 1998. "Developing student's writing skills in english through systematic grammar exercises and teachers's feedback". Kañina. 22 (2): 125-132.

Tardy, William. 1994. Composiciones ilustradas. Illinois: Nacional Textbook Company. Espéculo. Num. 15. Madrid: Universidad Complutense de Madrid.

Ur, Penny. 1995. A course in Language Teaching. Practice and theory. Cambridge: Cambridge University 